\title{
HEGRA Observations of Galactic Sources
}

\author{
Heinz Völk*, HEGRA Collaboration \\ * Max-Planck-Institut für Kernphysik \\ P.O. Box 103980, 69029 Heidelberg, Germany
}

\begin{abstract}
In this talk I will first give a summary of the observations of expected Galactic TeV $\gamma$-ray sources with the HEGRA CT-Sytem since the Kruger Park Workshop in 1997. Then I will go into some detail regarding the observations of Supernova Remnants (SNRs), especially those of Tycho's SNR and of Cas A. The emphasis will not be on all aspects of these published data. I will rather review the selection of these observational targets, and discuss some of the physical implications of the results.
\end{abstract}

\section{SUMMARY OF OBSERVATIONS}

The stereoscopic system of imaging atmospheric Cherenkov telescopes (IACTs) of HEGRA has been running since late 1996 with four telescopes. After a fire in the array which also damaged one of these telescopes late in the year 1997, the final configuration of five equal telescopes with identical cameras has become operational in August 1998. Apart from the IACT system, HEGRA successfully operates a stand-alone telescope, called CT1; it is also doing obervations during moon periods. However in this review, I will be concerned with the stereoscopic system alone.

Since 1997 a number of Galactic source candidates has been observed with the IACT system. The Galactic coordinates of the objects discussed in this paper are indicated in Figure 1. The objects analyzed are given in Table 1.

1. The observations of the Crab Nebula were done both at normal $\left(\mathrm{ZA} \leq 30^{\circ}\right)$ and at high zenith angles $\left(\mathrm{ZA} \sim 60^{\circ}\right)$. They led to a (combined) energy spectrum up to $20 \mathrm{TeV}$ (Konopelko et al. 1999). It is within the errors compatible with an extension of the power law spectrum inferred from measurements in the $\mathrm{TeV}$ energy range (Konopelko et al., these Proceedings). Thus no possible hard hadronic emission component has been identified up to these energies.

2. A search for a periodic signal from the Crab and Geminga Pulsars was also performed (Aharonian et al. 1999a). No evidence for pulsed emission was found. Even though we had expected Geminga to be a major contributor to the distribution 


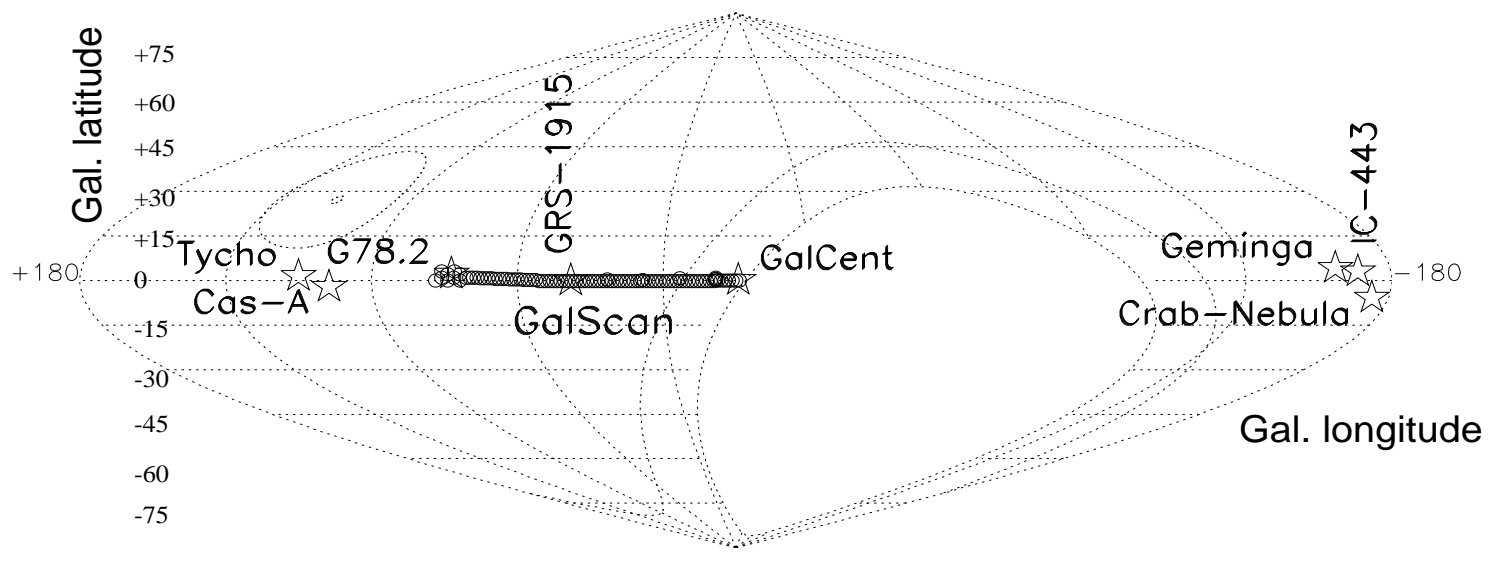

FIGURE 1. Part of the sky in Galactic coordinates. Shown are the positions of the objects discussed in this paper. The white part is not visible from La Palma; the band surrounding that region can only be observed under restricted conditions.

of very energetic CR electrons in the neighborhood the Solar System, it showed up as a $\mathrm{TeV}$-quiet object.

3. The observations of the Galactic Microquasar GRS 1915 have been analysed (Kettler 1999). No signals have been found during these observation periods. This is not too surprising since, unfortunately, the source had also been low in other wavelength ranges in those times.

4. The two SNRs Tycho and Cas A have been observed extensively with the stereoscopic system. This is especially true for Cas A, where a deep observation of $128 \mathrm{hr}$ duration has been included in the present analysis (Pühlhofer et al. 1999a). The two objects will be discussed in some detail in section 2 .

5. An extensive Galactic Plane scan ( $\geq 2$ hrs of observation time for each point,

TABLE 1. Galactic objects observed 1997-1999 with the HEGRA IACT-System. The numbers given are the observation hours in the respective years for the analysis of the Galactic Cosmic-Ray (CR) proton spectrum, CR background (bgr) events were used.

\begin{tabular}{|c|c|c|c|c|}
\hline Source & {$[1997]$} & [1998] & [1999] & References \\
\hline Crab & 92 & 138 & 31 & $\begin{array}{l}\text { Konopelko et al. 1999, } \\
\text { Aharonian et al. } 1999 \text { a }\end{array}$ \\
\hline Geminga & - & 23 & - & Aharonian et al. 1999a \\
\hline GRS 1915 & 50 & 12 & 11 & Kettler 1999 \\
\hline Cas A & 102 & 85 & - & Pühlhofer et al. 1999a \\
\hline Tycho & 23 & 35 & - & Pühlhofer et al. 1999a \\
\hline Gal. Plane & 111 & 66 & - & Pühlhofer et al. 1999b \\
\hline Diff. VHE & - & - & 53 & Lampeitl et al. 1999 \\
\hline CR Protons & bgr & bgr & bgr & Aharonian et al. 1999b \\
\hline
\end{tabular}




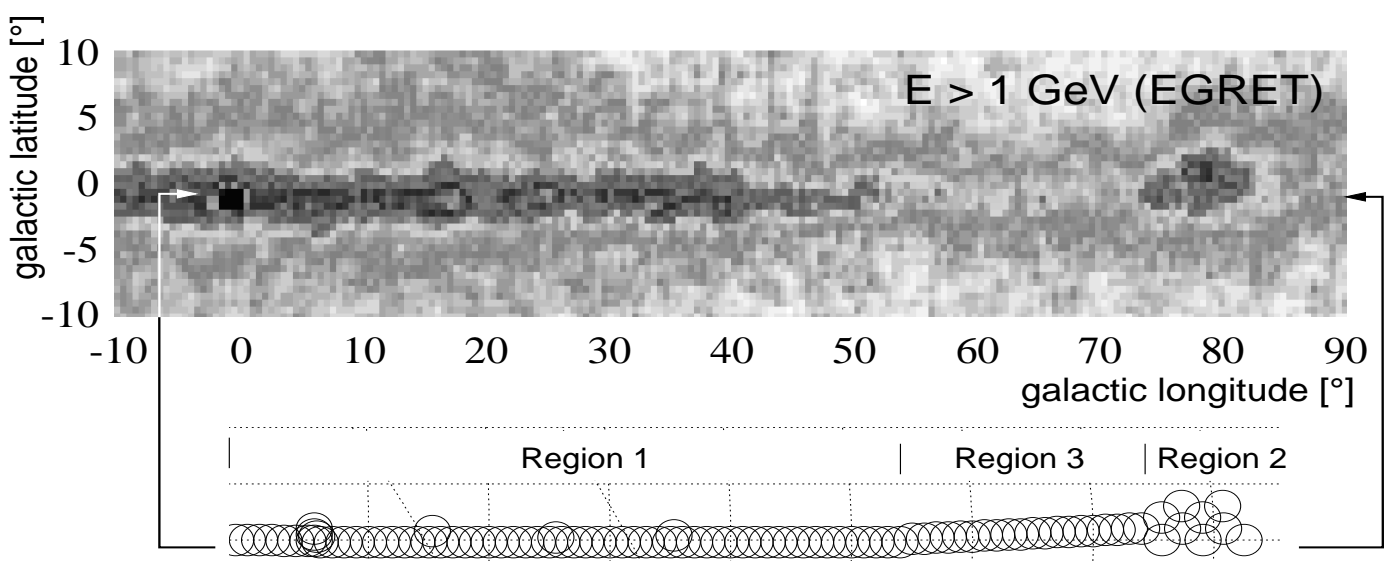

Culmination zenith angle:

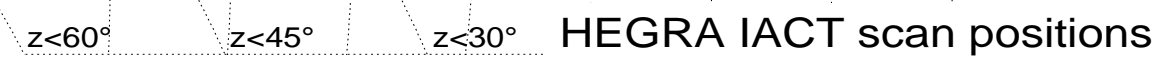

FIGURE 2. The HEGRA IACT scan positions in the Galactic Plane. For comparison also the corresponding part of the EGRET sky map for $\gamma$-ray energies $E>1 \mathrm{GeV}$ is shown. Regions 1 and 2 were observed in 1997, region 3 in 1998.

plus some re-observations) in the $\mathrm{TeV}$ band covered the Galactic longitude region from the Galactic Center $\left(l,-1.5^{\circ}\right)$ to the Cygnus region $\left(l, 83.5^{\circ}\right)$, see Figure 2 .

Sources with a flux above 1/4 Crab units should have been detected, as indicated by Table 2 below. A first analysis reveals no hints for such strong TeV point sources (Pühlhofer et al. 1999b).

6. In a similar vein, a program regarding the search for diffuse VHE $\gamma$-ray emission from the Galactic Plane was started. The present analysis is largely of a technical nature (Lampeitl et al. 1999). The observations will be continued during this summer of 1999.

7. Finally, the imaging Cherenkov technique was applied for the first time to the determination of the flux and the TeV energy spectrum of the charged CR protons. For this work background events for the Mkn 501 observations from 1997 were used. Calibration is exclusively by Monte Carlo simulations that include a detailed detector simulation. For physical reasons, the proton detection rate strongly exceeds that for heavier CR nuclei near threshold, around 1.5 TeV. The stereoscopic detection of the air showers permits the effective suppression of air showers induced by heavier particles already at the trigger level, and in addition by software analysis cuts. The results are in good agreement with the recent results of satellite and balloone-borne experiments and reach similar accuracy (Aharonian et al. 1999b). Without any knowledge of the CR composition, the proton spectrum can only be determined with precision near threshold. However, it should be possible to obtain in addition an approximate CR composition, using further specific image cuts (Plyasheshnikov et al. 1998). This will allow an extension of the dynamical 
range of the spectrum into an energy region that is very costly to cover by direct detection CR experiments. I think it would be important if in addition the large Zenith angle technique could be applied to this problem.

Of course, more than these objects have been observed in the Galaxy. However the data have not been analyzed yet, and are therefore not a subject of this summary.

Let me conclude this section with a general consideration.

As mentioned above, the HEGRA Galactic Plane scan has not yet led to the detection of new sources. In his excellent introductory review, Trevor Weekes (these Proceedings) described this result as "depressing".

We were also disappointed. On the other hand, the result is perhaps not too surprising, given the low sensitivity level with which this survey had to be done. The result should also prompt a new discussion about the aims and possibilities of ground-based $\gamma$-ray astronomy with imaging telescopes. Space is scarce in these proceedings. So, I will summarize my arguments only briefly in four points, and hope that they open a broader debate: (i) we should of course continue such surveys; any field of astronomy must do this (ii) however it is not too probable that we will find new sources that have not been seen as unusual objects in another wavelength range already, considering the enormous investments in ever more powerful instruments in the radio, infrared, optical, and X-ray domains that have been made over the last two decades (iii) thus, our main activity should perhaps be to look at sources also known in other wavelength-ranges; only then we can hope to obtain a physical understanding of the $\gamma$-ray results (iv) given the much higher physical complexity of the acceleration and transport processes for the nonthermal component than for the thermal component, the potential for discovery is one for strong nonthermal activity in known objects, and it is as important as the potential for discovery of previously unknown objects in the more conventional "thermal" astronomy.

I do not believe that the $\gamma$-ray bursts provide a counter argument to this point of view: they are explosive events in previously inconspicuous objects, and could not have been found in a survey with a narrow-FoV instrument like IACTs; an all-sky capability was needed to discover them, and they were difficult to understand for decades before they were detected also in other wavelength ranges. Also Geminga, originally an enigmatic Cos B source, is not really a counterexample, because Geminga could only be physically identified after many years, when ROSAT

discovered that it was a long-period Pulsar and determined its period, which was subsequently confirmed by EGRET.

\section{SUPERNOVA REMNANTS}

\section{Observations}

Earlier observations of the SNRs G87.2+2.1 ( $\gamma$ Cygni) and IC 443 in 1996/97 gave consistent upper limits between Whipple (Buckley et al. 1998) and the HEGRA 
CT-System (Heß 1998) at effective threshold energies, for the Zenith angles involved, of $E_{\gamma}>300 \mathrm{GeV}$ and $E_{\gamma}>800 \mathrm{GeV}$, respectively. They were slightly above theoretical predictions regarding the $\pi^{0}$-decay $\gamma$-ray emission for a uniform ISM but well within astronomical uncertainties (Völk 1997). Both objects had originally been assumed to interact with interstellar clouds. Under ideal assumptions such an interaction could have increased the $\pi^{0}$-decay $\gamma$-ray luminosity significantly. These two SNRs are presumably the result of core collapse Supernovae, due to massive $\left(M>8 \mathrm{M}_{\odot}\right)$ progenitor stars. If they have masses exceeding roughly $15 M_{\odot}$, these stars have stellar winds which significantly modify the circumstellar environment. For such "Wind-SNe" the time history of the $\pi^{0}$-decay $\gamma$-ray emission is much more complex: except within the wind zone, it is much lower than for a uniform ISM of the same density (Berezhko \& Völk 1995, Berezhko \& Völk 1997).

The recent HEGRA observations concern deep observations of Tycho's SNR, believed to be a SN Ia in a uniform ISM with strong X-ray lines and no or only a very weak nonthermal X-ray continuum, and of Cas A, assumed to be a SN Ib resulting from the core collapse of a very massive Wolf-Rayet star, with a strong nonthermal X-ray continuum - an archetypical Wind-SN (Pühlhofer et al. 1999a). Both SNRs are very young in an evolutionary sense, presumably still in the sweepup phase, even though this might only be marginally true for Tycho's SNR.

The long observation times which we have reserved for these objects imply a change in our ideology: the emphasis is no more on SNR shocks that presumably interact with interstellar clouds, but on very young objects, either in a supposedly uniform ISM or in a strongly modified precursor wind structure.

\section{Data analysis for Cas A and Tycho}

The following data analysis for Tycho and Cas A has been done by G. Pühlhofer. For the angular resolution of the HEGRA CT-system of 0.05 to $0.1^{\circ}$ Tycho is a

$\gamma$-ray point source, and Cas A is marginally extended. Due to the available Zenith angles the instrument threshold is at $1 \mathrm{TeV}$ or slightly above (see Table 2). The complete sensitivities of the IACT system are described in the article by M. Panter (these Proceedings). The observation times and significances are given in Table 2.

With $\sim 38$ hrs of observation no signal has yet been found from Tycho, whereas the full data sample of $\sim 128 \mathrm{hrs}$ for Cas A shows evidence for a signal above 1 $\mathrm{TeV}$. For Cas A the event statistics as a function of (distance) ${ }^{2}$ is shown in the left panel of Figure 3 , both for a point source assumption (I), and for a slightly extended source (II). The position of the $\gamma$-ray source on the sky is given in the right panel, and is consistent with the radio astronomical position that corresponds to the center of the picture.

Figure 4 shows a model calculation for the energy spectrum of Cas A (Atoyan et al. 1999b). The full and the dashed lines correspond to the expected inverse Compton (IC) emission, as derived phenomenologically from the observed synchrotron 


\begin{tabular}{|c|c|c|c|c|}
\hline & \multicolumn{2}{|c|}{ Tycho's SNR } & \multicolumn{2}{|c|}{ Cas-A } \\
\hline Configuration: & " time & $\begin{array}{l}\text { mean zenith } \\
\text { angle / } E_{\text {thr }}\end{array}$ & time & $\begin{array}{l}\text { mean zenith } \\
\text { angle / } \mathrm{E}_{\text {thr }}\end{array}$ \\
\hline 1: 1997 CT 3-6 & $20.8 \mathrm{hrs}$ & $\vartheta=37^{\circ}$ & $49.3 \mathrm{hrs}$ & $\vartheta=31^{\circ}$ \\
\hline 2: 1997 СТ 3,5,6 & & & $20.0 \mathrm{hrs}$ & $\vartheta=32^{\circ}$ \\
\hline 3: 1998 CT 3-6 & $16.8 \mathrm{hrs}$ & $\vartheta=36^{\circ}$ & $58.6 \mathrm{hrs}$ & $\vartheta=33^{\circ}$ \\
\hline Total & $37.6 \mathrm{hrs}$ & $\mathrm{E}_{\mathrm{thr}} \approx 1.2 \mathrm{TeV}$ & $127.9 \mathrm{hrs}$ & $\mathrm{E}_{\mathrm{thr}} \approx 1 \mathrm{TeV}$ \\
\hline $\begin{array}{l}\text { significance above background } \\
\text { (probability cut) }\end{array}$ & & $-1.1 \sigma$ & & $4.5 \sigma$ \\
\hline
\end{tabular}

FIGURE 3. Observation times and significances for Tycho's SNR and Cas A; the 37.6 hrs for Tycho constitute only part of the total observation time available. The stereoscopic configuration of the four sytem telescopes CT 3-6, used for these observations, was different for the second 1997 period due to the fire on La Palma that hit CT 4. For the analysis of Cas A the source was assumed to be slighly extended.

spectrum from the radio to the hard X-ray region (Atoyan et al. 1999a), for the two mean magnetic field strengths $B_{1}=1 \mathrm{mGand}=1.6 \mathrm{mG}$, respectively. The heavily dotted curve corresponds to a $\pi^{0}$-decay spectrum, produced by an assumed power law spectrum of protons accelerated in the source, with a total energy content of $W_{p}=2 \times 10^{49} \mathrm{ergs} \simeq 1 / 5 W_{p}(t=\infty)$, where $W_{p}(t=\infty)=10^{50} \mathrm{erg}$ corresponds to an assumed time-asymptotic nonthermal fraction of 10 percent of the total hydrodynamic energy of $10^{51} \mathrm{erg}$, generally assumed to be released in Cas A. The proton spectral index assumed is 2.15 , with a rather high cutoff at $200 \mathrm{TeV}$ for this Wind-SN already at very early times (Völk \& Biermann 1988). The mean thermal gas density seen by the relativistic protons is taken as $15 \mathrm{~cm}^{-3}$. The energetic electrons responsible for the IC emission are assumed to come from three different regions of the SNR interior: the bright compact radio components with magnetic field strength $B_{1}=1 \mathrm{mG}$, where electrons are accelerated locally, an extended "plateau" of shocked circumstellar gas with $B_{2}=B_{1} / 4$ due to global acceleration at the forward SNR shock, and a low-field part of this "plateau" with $B_{3}=0.1 \mathrm{mG}$. The electron spectral index is assumed to be uniformly 2.15, as for the protons. The electron cutoff energy, however, is only $17 \mathrm{TeV}$, corresponding to the steep drop-off of the observed hard X-ray spectrum with increasing energy.

Clearly, the magnitude of the $\gamma$-ray flux at about $1 \mathrm{TeV}$, if ultimately detected with a significance exceeding $5 \sigma$, could be equally due to electronic IC or hadronic $\pi^{0}$-decay emission. However the spectra would be very different for the two cases: an IC spectrum should fall off strongly with energy, in contrast to a $\pi^{0}$-decay spectrum. Therefore I believe that every effort should be made to obtain a TeVspectrum of Cas A.

Acknowledgements I am grateful to Gerd Pühlhofer for providing several of 

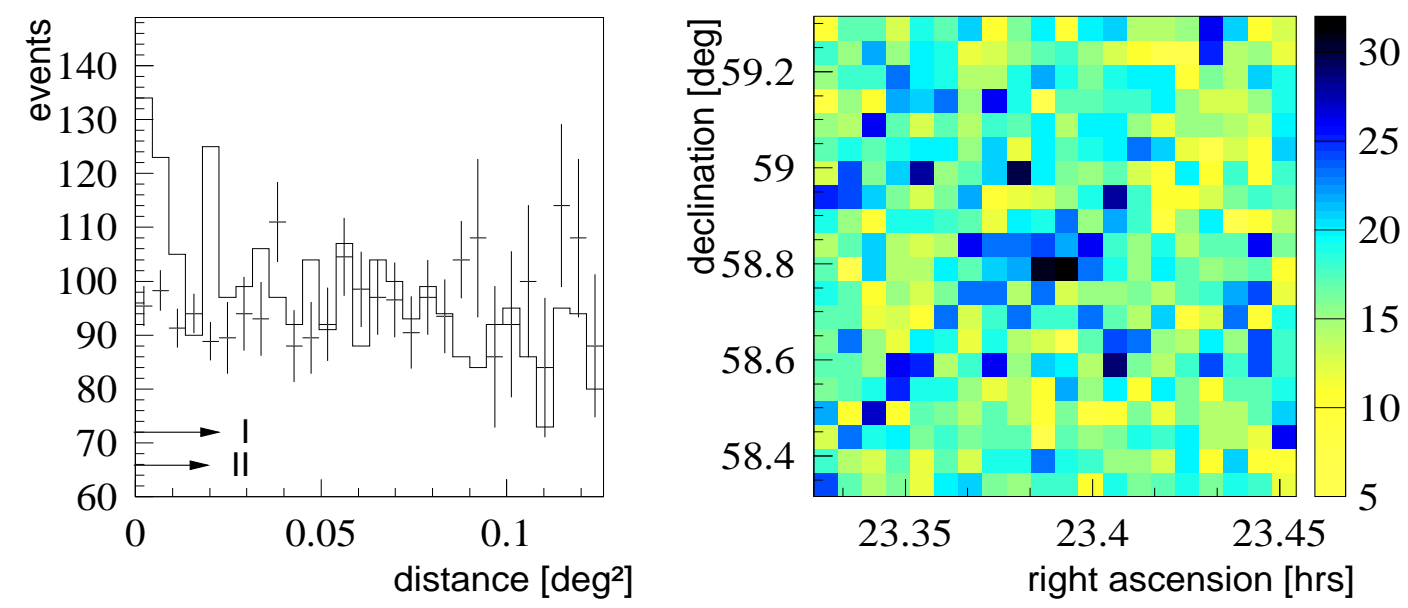

FIGURE 4. Event statistics and position determination of the $\gamma$-ray source Cas A. See text for details.

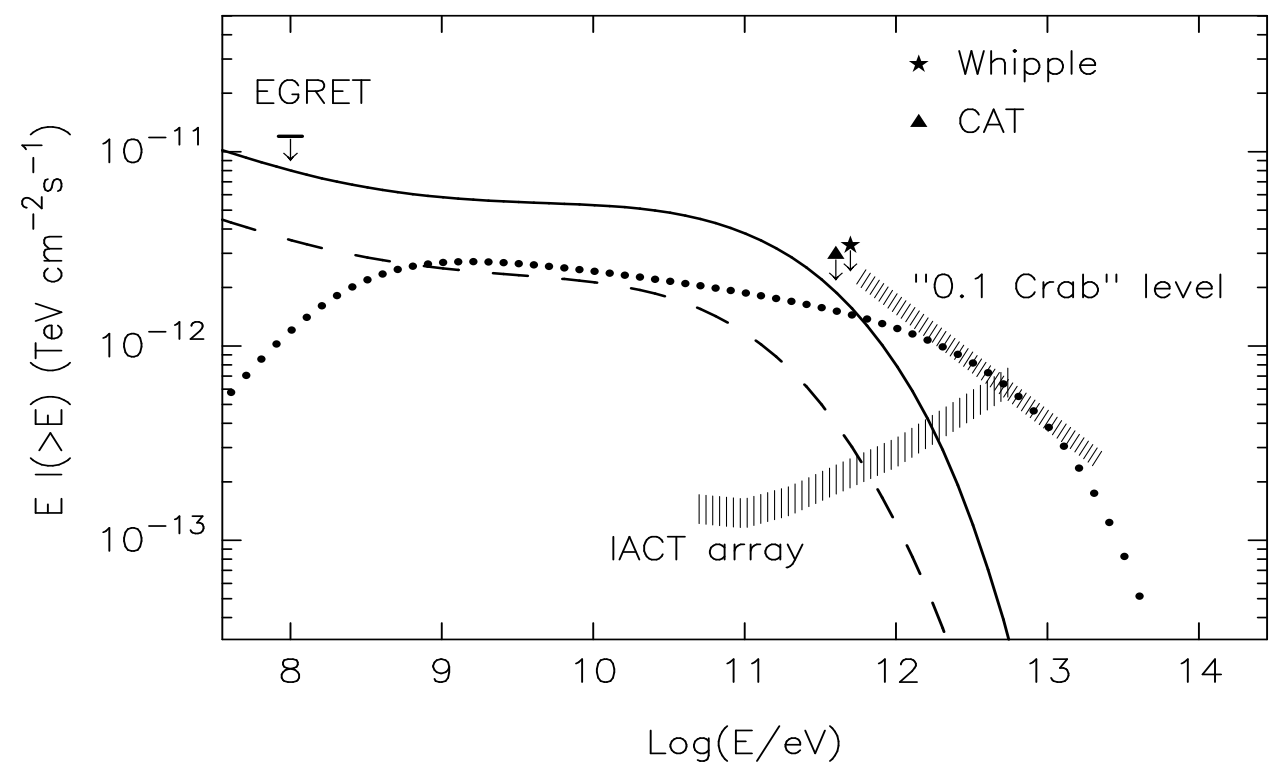

FIGURE 5. Energy flux spectrum of Cas A in $\gamma$-rays . The full and the dotted curve correspond to the inferred IC emission, for a magnetic field strength $B_{1}$ in the bright compact radio knots, given by $B_{1}=1 \mathrm{mG}$ and $=1.6 \mathrm{mG}$, respectively. The heavily dotted curve is an assumed $\pi^{0}$-decay spectrum thought to be appropriate for the present evolutionary state of the remnant (see text). Indicated are also the upper limits reported by the Whipple (Lessard et al. 1999) and CAT collaborations (Goret et al. 1999). The slantedly hatched curve denotes 1/10 of the Crab flux. The vertically hatched curve corresponds to the sensitivity of the future arrays Cangaroo III, H.E.S.S. and VERITAS. 
the Figures in this paper.

\section{REFERENCES}

1. Konopelko, A.K., Pühlhofer, G., et al., Proc. 26th ICRC, Salt Lake City 3, 444 (1999).

2. Aharonian, F.A., Akhperjanian, A.G., Barrio, J.A., et al., A $\& A$ 346, 913 (1999a).

3. Kettler, J., private communication, (1999).

4. Pühlhofer, G., Völk, H.J., Wiedner, C.-A., et al., Proc. 26th ICRC, Salt Lake City 3, 492 (1999).

5. Pühlhofer, G., Bernlöhr, K., Daum, A., et al., Proc. 26th ICRC, Salt Lake City 4, 77 (1999).

6. Lampeitl, H., Konopelko, A.K., et al., Proc. 26th ICRC, Salt Lake City 4, 81 (1999).

7. Aharonian, F.A., Akhperjanian, A.G., Barrio, J.A., et al., A\&A 59, 092003-1 (1999b).

8. Plyasheshnikov, A.V., Konopelko, A.K., Aharonian, F.A., et al., J. Phys. G 24, 653 (1998).

9. Buckley, J.H., Akerlof, C.W., Carter-Lewis, D.A., et al., A\&A 329, 639 (1999).

10. Heß, M., PhD Thesis Univ. Heidelberg (1998).

11. Völk, H.J., Proc. "Towards a Major Atmospheric Cherenkov Detector V", Kruger Park, S.A., 87 (1997).

12. Berezhko, E.G., Völk, H.J., Proc. 24th ICRC, Rome 3, 380 (1995).

13. Berezhko, E.G., Völk, H.J., Astroparticle Phys. 7, 183 (1997).

14. Atoyan, A.M., Aharonian, F.A., Tuffs, R.J., et al., $A \mathscr{E} A$ in press (1999a).

15. Atoyan, A.M., Aharonian, F.A., Tuffs, R.J., et al., $A \mathscr{E} A$ submitted to $A \mathscr{E} A$ (1999b).

16. Völk, H.J., Biermann, P.L., ApJ 333, L65 (1988).

17. Lessard, R.W., Bond, I.H., Boyle, P.J., et al., Proc. 26th ICRC, Salt Lake City 3, 488 (1999).

18. Goret, P., Guiffes, C., Nuss, E., et al., Proc. 26th ICRC, Salt Lake City 3, 496 (1999). 